\title{
Danshen Extracts Prevents Obesity and Activates Mitochondrial Function in Brown Adipose Tissue
}

\author{
Yoon Hee Cho, Cheol Ryong Ku, Young-Suk Choi, Hyeon Jeong Lee, Eun Jig Lee \\ Division of Endocrinology and Metabolism, Department of Internal Medicine, Yonsei University College of Medicine, Seoul, \\ Korea
}

Background: Danshen has been widely used in oriental medicine to improve body function. The purpose of this study is to investigate the effect of water-soluble Danshen extract (DE) on weight loss and on activation proteins involved in mitochondrial biogenesis in brown adipose tissue (BAT) in obese mice.

Methods: BAT was isolated from 7-week-old male Sprague-Dawley rats, and expression of proteins related to mitochondrial biogenesis was confirmed in both brown preadipocytes and mature brown adipocytes treated with DE. For the in vivo study, low-density lipoprotein receptor knock out mice were divided into three groups and treated for 17 weeks with: standard diet; high fat diet (HFD); HFD+DE. Body weight was measured every week, and oral glucose tolerance test was performed after DE treatment in streptozotocin-induced diabetic mice. To observe the changes in markers related to thermogenesis and adipogenesis in the BAT, white adipose tissue (WAT) and liver of experimental animals, tissues were removed and immediately frozen in liquid nitrogen.

Results: DE increased the expression of uncoupling protein 1 and peroxisome proliferator-activated receptor gamma coactivator 1-alpha in brown preadipocytes, and also promoted the brown adipocyte differentiation and mitochondrial function in the mature brown adipocytes. Reactive oxygen species production in brown preadipocytes was increased depending on the concentration of DE. DE activates thermogenesis in BAT and normalizes increased body weight and adipogenesis in the liver due to HFD. Browning of WAT was increased in WAT of DE treatment group.

Conclusion: DE protects against obesity and activates mitochondrial function in BAT.

Keywords: Adipose tissue, brown; Organelle biogenesis; Dan-shen root extract; Thermogenesis; Obesity

\section{INTRODUCTION}

Obesity increases risks of metabolic complications such as insulin resistance and type 2 diabetes [1-3]. Obesity, caused by the imbalance between energy intake and energy expenditure, reduces the biogenesis and function of mitochondria in brown fat [4-6]. Brown adipose tissue (BAT) is important for energy ex-

Received: 2 September 2020, Revised: 29 December 2020,

Accepted: 31 December 2020

Corresponding author: Eun Jig Lee

Division of Endocrinology and Metabolism, Department of Internal Medicine, Yonsei University College of Medicine, 50-1 Yonsei-ro, Seodaemun-gu, Seoul 03722, Korea

Tel: +82-2-2227-8129, Fax: +82-2-393-6884, E-mail: ejlee423@yuhs.ac penditure and when it receives a stimulus, BAT can convert a large amount of calories into heat by mitochondrial uncoupling and uncoupling protein 1 (UCP1) is key role in the thermogenesis program [7].

Peroxisome proliferator-activated receptor gamma coactivator 1-alpha (PGC-1 $\alpha)$ is known to regulate mitochondrial biogenesis and the brown fat differentiation in BAT, as well as is

\section{Copyright $(\odot 2021$ Korean Endocrine Society}

This is an Open Access article distributed under the terms of the Creative Commons Attribution Non-Commercial License (https://creativecommons.org/ licenses/by-nc/4.0/) which permits unrestricted non-commercial use, distribution, and reproduction in any medium, provided the original work is properly cited. 
known to enhance the UCP1 expression in the brown fat by interacting with peroxisome proliferator-activated receptor $\gamma$ $(\operatorname{PPAR} \gamma)$ [7-10]. Notably, in mice deficient in PGC-1 $\alpha$, the expression of UCP1 is reduced, so PGC- $1 \alpha$ plays an essential role in adaptive thermogenesis in cold environments [11]. The activation of AMP-activated protein kinase (AMPK) was known to directly affect the PGC- $1 \alpha$ activity through the phosphorylation [12]. The activity of AMPK in adipocytes is known to play an important role in regulating the browning process from white adipose tissue (WAT) to BAT and maintaining energy homeostasis [13].

Commonly used anti-diabetic drugs can also induce AMPK activity in adipocytes, supporting the observation that AMPK mediates metabolic regulation for the control of whole-body energy homeostasis [14]. Therefore, promoting BAT function through the activation of PGC-1 $\alpha$ and AMPK has therapeutic potential for obesity [15].

Danshen (Salvia miltiorrhiza) is a traditional Chinese herb for the treatment of many diseases, improving microcirculation, vasodilation, anticoagulation, and anti-inflammation [16,17]. Danshen can be divided into water-soluble agents or lipid-soluble one, depending on solubility. Most studies on the effects of Danshen has been reported on lipid-soluble components, such as tanshinone IIA [18]. The beneficial effects of Danshen have been reported in other many studies; however, the effect of water-soluble Danshen on the treatment of obesity through the mitochondrial activity in BAT has not yet been evaluated.

To evaluate the therapeutic effects of a water-soluble Danshen extract (DE) on mitochondrial function in BAT and browning processing of WAT, we used isolated adipocyte from BAT and high fat diet (HFD)-induced obese mouse models. In the HFD animal model, we investigated the expression of markers related to thermogenesis in BAT and WAT. In addition, the anti-diabetic effect of DE in the streptozotocin (STZ)-induced diabetes model was evaluated.

\section{METHODS}

\section{Ethics statement}

All animal experimental protocols were reviewed and approved by the Institutional Animal Care and Use Committee (IACUC) of the Yonsei Laboratory Animal Research Center (YLARC) (Permit \#: 2010-0268). All animal studies were performed in facilities approved by the Association for Assessment and Accreditation of Laboratory Animal Care. Experimental protocols were reviewed and approved by the IACUC of the YLARC (permit No.: 2010-0268).

\section{Production of Danshen extracts}

DE has been provided by the Dalim Biotec (Seoul, Korea). Briefly description, dried and powdered roots of S. miltiorrhiza (200 g) were boiled with water $(1,600 \mathrm{~mL})$ for 2 hours. The extracted solution was then filtered and solid deposits were removed. The extracted solution was then concentrated by heating with a rotary evaporator. Next, identical volumes of extracted solution and butanol were mixed in a separatory funnel. The upper solution of the mixture was then collected and concentrated with a rotary evaporator. The components of the collected DE were evaluated via ultra performance liquid chromatographyultraviolet (UPLC-UV). Powdered DE was dissolved in distilled water containing 1\% dimethyl sulfoxide (DMSO) (SigmaAldrich Co., St. Louis, MO, USA) and diluted to final experimental concentrations.

\section{Experimental animals and diets}

Fifteen low-density lipoprotein receptor (LDLR) knock out male mice were housed in specific pathogen-free conditions, with a temperature of $21^{\circ} \mathrm{C} \pm 2.0^{\circ} \mathrm{C}$, relative humidity of $50 \% \pm$ $5 \%$, and a 12-hour-light/12-hour-dark cycle. From 1 week before the dietary intervention, all animals were provided free access to commercial rodent food and tap water. At the beginning of the study, 10 weeks old mice were divided into three groups: (1) control group fed a normal diet (placebo; $n=5$ ); (2) a group fed the HFD ( $60 \%$ of calories from fat:high fat $[\mathrm{HF}] ; n=5)$; and (3) a group fed the HFD administered orally with $50 \mathrm{mg} / \mathrm{kg}$ of $\mathrm{DE}(\mathrm{wt} / \mathrm{wt})(\mathrm{HF}+\mathrm{DE} ; n=5)$. Body weight and food intake were monitored throughout the study. After 17 weeks, experimental animals were sacrificed. Tissues were snap-frozen immediately in liquid nitrogen or fixed with $4 \%$ paraformaldehyde and stored at $-80^{\circ} \mathrm{C}$ until further processing. $\mathrm{DE}$ has been provided by the Dalim Biotec.

For the diabetes animal models, C57/BL6J mice were divided into the following three groups: normal control group (ctrl; $n=8$ ), diabetic group (STZ; $n=12$ ), and diabetic group treated with DE extract $(\mathrm{STZ}+\mathrm{DE} ; n=12)$. For the experiment, 10 -week-old mice in the STZ and experimental groups were starved for 16 hours and $75 \mathrm{mg} / \mathrm{kg}$ STZ (Sigma-Aldrich) was intraperitoneally injected daily for three consecutive days. STZ powder was freshly diluted in $0.1 \mathrm{M}$ sodium citrate buffer $(\mathrm{pH}$ 4.5 ) immediately before injection. The experimental group was orally administered DE extract $(50 \mathrm{mg} / \mathrm{kg}$ ) for 1 week after confirming diabetes induction. The control group was injected with 
only $0.1 \mathrm{M}$ sodium citrate buffer. Blood glucose concentration in mice were measured daily before STZ injection and every 2 to 4 days after three consecutive STZ injections. After 13 days of the first STZ injection, the mice were starved for 16 hours and an oral glucose tolerance test (OGTT) was performed by the oral administration of $2 \mathrm{~g} / \mathrm{kg}$ glucose. The blood glucose concentration was measured at $0,30,60$, and 120 minutes after the administration of $2 \mathrm{~g} / \mathrm{kg}$ glucose using the tail nick bleeding method with a glucometer (Arkray, Minneapolis, MN, USA).

\section{Primary cell isolation and culture}

Brown preadipocytes were isolated from the interscapular BAT of 7-week-old male Sprague-Dawley rats (Orient Bio Inc., Seongnam, Korea) by collagenase digestion and were differentiated as described previously [19,20]. Briefly, isolated brown preadipocytes were incubated in high glucose Dulbecco's Modified Eagle's Medium (DMEM) containing 1\% antibiotics solution and 10\% fetal bovine serum (FBS; Gibco-Invitrogen, Bethesda Research Laboratories, Waltham, MA, USA) at $37^{\circ} \mathrm{C}$ in a humidified atmosphere with $5 \% \mathrm{CO}_{2}$. DE was treated for 24 hours at different concentration in brown preadipocytes, and then each protein was obtained. For differentiation, primary cultured brown adipocytes were grown in DMEM supplemented with 10\% FBS, $1 \mathrm{nM} \mathrm{T3}$, and $20 \mathrm{nM}$ insulin (differentiation medium $[\mathrm{DM}]$ ) until reaching $70 \%$ confluence for 2 days. The cells were then cultured in DM supplemented with $0.5 \mathrm{mM}$ isobutyl-lmethylxanthine, and $0.15 \mu \mathrm{M}$ dexamethasone (induction medium [IM]) for 2 days. Next, the cells were cultured in DM until they exhibited a fully differentiated phenotype with multiple multi-lobular lipid droplets in the cytoplasm. For the in vitro study, DE was administered during the IM supplement period and maintained thereafter.

\section{Cell proliferation assay}

The effect of DE on cell proliferation and viability was assessed by using MTS assay (CellTiter 96 Aqueous One Solution Cell Proliferation Assay Kit, Promega, Madison, WI, USA). First, brown preadipocytes were incubated for 2 days in the presence of various concentrations of DE on 96 well plate. Later, cell viability was measured by enzyme-linked immunosorbent assay.

\section{Reactive oxygen species assay}

The effect of DE on intracellular reactive oxygen species (ROS) was examined as previously described. Briefly, synchronized brown preadipocytes were pretreated with DE (50 and $100 \mu \mathrm{g} /$ $\mathrm{mL}$ ) for 24 hours. Cells were then incubated for 30 minutes with CM-H $\mathrm{H}_{2} \mathrm{DCF}-\mathrm{DA}$ (Invitrogen Molecular Probes, Eugene, Oregon, USA) in Dulbecco's phosphate buffered saline (DPBS). Finally, CM- $\mathrm{H}_{2}$ DCF-DA emission was recorded using a fluorescence cytometer (Becton Dickinson, FACSCalibur, BD Biosciences, Franklin Lakes, NJ, USA). Samples were analyzed with FACSCalibur.

\section{Oil Red O staining}

At day 8 after differentiation, brown adipocytes were fixed with $4 \%$ formalin in phosphate buffered saline for 15 minutes and washed with distilled water. Cells were stained with filtered with $0.3 \%$ Oil Red O (Sigma \# O-0625) solution for 1 hour at room temperature. Stained cells were washed three times with distilled water. After completely drying at room temperature, phenotypic changes of adipogenic differentiation were observed using an inverted phase-contrast microscope (Olympus CKX41, Olympus, Tokyo, Japan).

\section{Western blot analysis}

Whole-cell lysates from frozen tissues and brown adipocytes were isolated using radioimmunoprecipitation assay (RIPA) lysis buffer (150 mmol/L Tris-HCl, $50 \mathrm{mmol} / \mathrm{L} \mathrm{NaCl}, 1 \% \mathrm{NP}-40$, $0.1 \%$ Tween-20). Protease and phosphatase inhibitors were added to all buffers before experiments. Western blot was performed as previously described [21]. Protein concentrations were assayed using a Quick Start ${ }^{\mathrm{TM}}$ Bradford Assay (Bio-Rad, Hercules, CA, USA). The primary antibodies anti-phosphorylated AMPK (p-AMPK) antibody (Cell Signaling, Danvers, MA, USA), anti-PPAR $\gamma$ antibody (Santa Cruz Biotechnology, Santa Cruz, CA, USA), anti-PGC-1 $\alpha$, and anti-UCP1 antibody (Abcam, Cambridge, UK) were incubated overnight at $4^{\circ} \mathrm{C}$, and specific proteins were visualized by the WESTSAVEup ${ }^{\mathrm{TM}}$ Detection system (AbFrontier, Seoul, Korea). Band intensities were measured using the GeneTool (SynGene, Cambridge, UK) and normalized to $\beta$-actin.

\section{Mitotracker staining and cell counting}

In brown preadipocytes, DE was treated for 24 hours and then, tumor necrosis factor- $\alpha$ (TNF- $\alpha ; 100 \mathrm{ng} / \mathrm{mL}, 1$ hour) treatment. To visualize mitochondrial distribution, brown adipocytes were stained with the mitochondrial marker Mitotracker Red CMH2XRos (Mito-Red, Molecular Probes) according to the manufacturer's instructions. Mito-Red was dissolved in a 1:1 mixture of dimethylsulfoxide and saline to a final concentration of 100 $\mathrm{nM}$. 


\section{RNA isolation and polymerase chain reaction analysis}

Total RNA was isolated from cultured brown adipocytes using Isol-RNA Lysis Reagent (5 PRIME, Gaithersburg, MD, USA) according to the manufacturer's instructions. First-strand cDNA synthesis from $1 \mu \mathrm{g}$ total RNA was performed using ReverTra Ace qPCR RT Kit (TOYOBO, Osaka, Japan). Quantitative realtime polymerase chain reaction (RT-PCR) reactions were performed as described [22]. Calculations were performed by a comparative method using glyceraldehyde 3-phosphate dehydrogenase $($ Gapdh) as an internal control. The primer sequences used in reverse transcription PCR were as follows: Gapdh: 5'-CAAGGTCATCCATGACAACT-3', 5'-TTCCCACCTTCTTGATGTC-3', PR domain containing 16 (Prdm16): 5'-CCTAGCCCTGAGCGATACTGTGA-3', 5'-ACAGACAATGGCTGGAATGGTG-3', UCP1: 5'-ACTGCCACACCTCCAGTCATT-3', 5'-CTTTGCCTCACTCAGGATTGG-3', Cidea: 5'-CTGTCTCAATGTCAAAGCCACGA-3', 5'-TGTGCAGCATAGGACATAAACCTCA-3', Ppar $\gamma$ : 5'-AGGTCAGAGTCGCCCCG-3', 5'-CCACAGAGCTGATTCCGAAG-3'.

\section{Statistics}

Data are expressed as mean \pm standard error. Statistical analyses were performed with the Student's $t$ test (two groups). $P<0.05$ was considered statistically significant.

\section{RESULTS}

\section{Danshen extract increases the expression of mitochondrial biogenesis-related proteins in brown preadipocytes}

To determine the appropriate concentration of DE on brown adipocyte differentiation, cytotoxicity was examined at 50, 100, and $500 \mu \mathrm{g} / \mathrm{mL}$ of DE in brown preadipocytes using the MTS assay. The viability of brown preadipocytes was not affected in 50 and $100 \mu \mathrm{g} / \mathrm{mL}$ concentrations of DE (Fig. 1A). Therefore, the treatment concentration of DE in brown adipocytes was determined as 50 and $100 \mu \mathrm{g} / \mathrm{mL}$. To investigate the effects of DE on the mitochondrial biogenesis of brown preadipocytes, the protein expression for UCP1, p-AMPK, and PGC-1 $\alpha$ was evaluated by Western blot analysis (Fig. 1B). DE did not affect the expression of UCP1 in preadipocytes, although DE increased its expression in differentiated brown adipocytes in a dose-dependent manner (Fig. 2C). DE increased AMPK activity and PGC$1 \alpha$ expression in brown preadipocytes. These results suggest that DE induces UCP1 expression and AMPK activation in mature brown adipocytes by inducing PGC- $1 \alpha$ expression in pre brown adipocytes. Importantly, the expression of PGC-1 $\alpha$ was significantly increased in brown preadipocytes treated with DE, suggesting that DE regulates brown adipose formation by activating thermogenesis and mitochondrial biogenesis.

Whether DE affects the number of mitochondria in brown preadipocytes was confirmed using Mito Tracker Red 580. When treated with DE, mitochondria tended to increase, but there was no significance. In brown preadipocytes, TNF- $\alpha$ (100 $\mathrm{ng} / \mathrm{mL}, 1$ hour) treatment significantly reduced the number of mitochondria, but in the pretreated group of DE, TNF- $\alpha$ induced mitochondrial reduction was not observed (Fig. 1C).

Stress increases ROS in adipocytes, thereby regulating mitochondrial biogenesis and UCP1 leading to thermogenesis. As shown in Fig. 1D, ROS production in brown preadipocyte was significantly increased depending on the concentration of DE.

\section{Danshen extract promotes brown adipocyte differentiation and mitochondrial function}

In order to study the roles of DE in the differentiation of brown adipocytes, various concentrations of DE were used for treatment until 2 days before differentiation (Fig. 2A). It was confirmed that the differentiation of brown adipocytes increased depending on the concentration of DE by using the Oil Red O staining. However, it is thought that the differentiation of brown preadipocytes is not induced at the concentration of DE $200 \mu \mathrm{g}$ due to the toxicity of the cells by the high concentration of the DE.

Because CCAAT/enhancer binding proteins (C/EBPs) are important components of adipocyte differentiation events, we investigated whether $\mathrm{DE}$ affects $\mathrm{C} / \mathrm{EBPs}$ expression in brown adipocytes. As shown the Fig. 2B, DE trended to increase the expression of $\mathrm{C} / \mathrm{EBP} \alpha$ and $\mathrm{C} / \mathrm{EBP} \beta$ in differentiated brown adipocytes, but this was not significant. The expression of UCP1 was also increased according to the pattern of increase in $\mathrm{C} / \mathrm{EBP} \alpha$ and C/EBP $\beta$ when treated with DE (Fig. 2C). Since the upregulation of UCP1 protein in brown fat is closely related to the adaptive heat generation activity and energy expenditure, it can be predicted that the increase of UCP1 expression by the treatment of DE will activate thermogenic program.

As shown in Fig. 2C, DE also increased the expression of PGC-1 $\alpha$ and AMPK activity in brown adipocytes, as a result, DE enhanced mitochondrial biogenesis during the brown adipogenesis. These results indicated that DE increased the differentiation of brown adipocytes accompanied by mitochondrial biogenesis, enhancing the mitochondrial function for the uncoupling of respiration. 

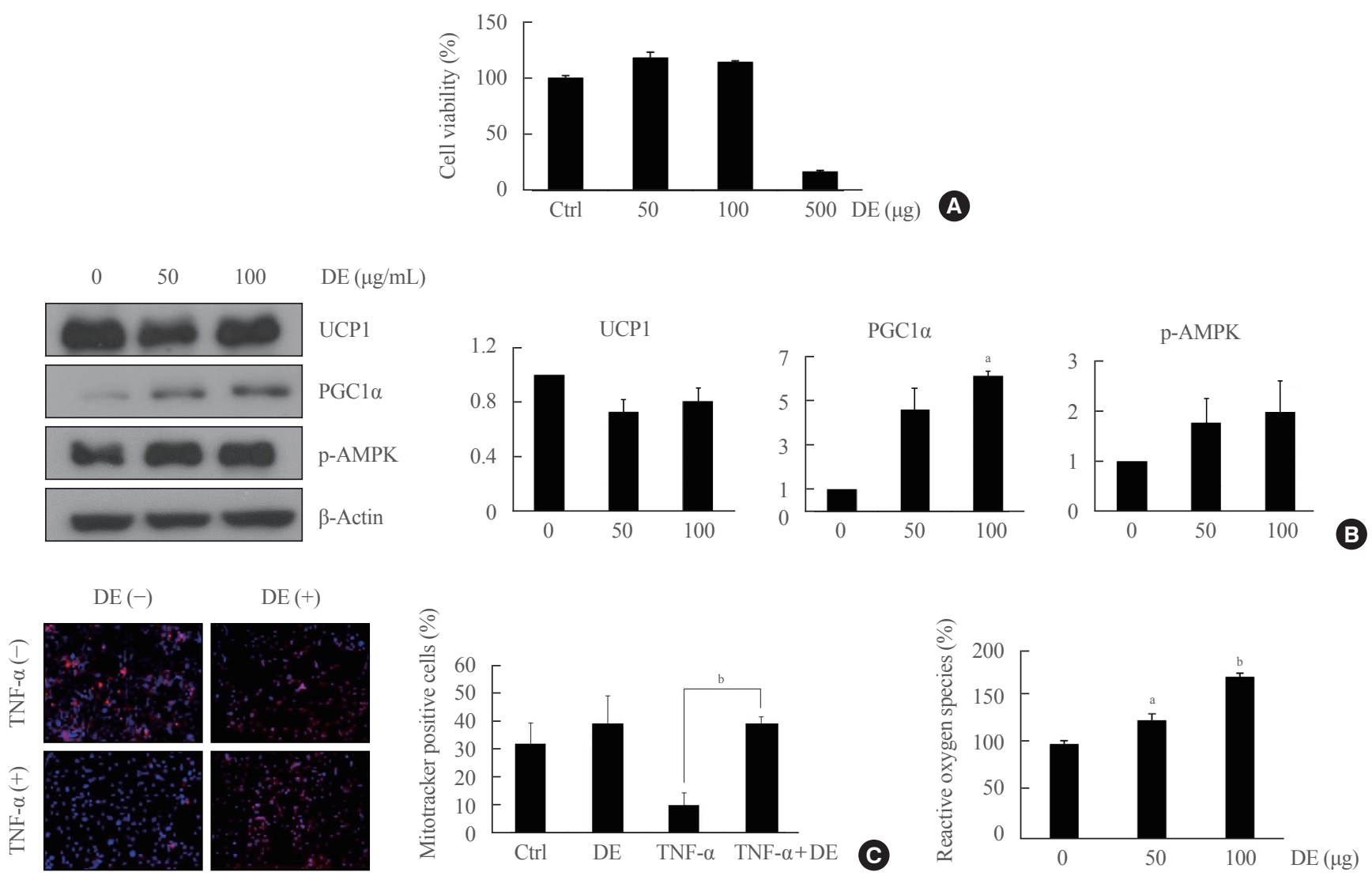

Fig. 1. Danshen extracts (DEs) increase the expression of mitochondrial biogenesis-related proteins in brown preadipocyte. (A) The effect of DE on cell proliferation and viability was assessed by using MTS assay. Brown preadipocytes were incubated for 2 days in the presence of various concentrations of DE on 96 well plate. Later, cell viability was measured by enzyme-linked immunosorbent assay. (B) Western blotting analysis of uncoupling protein 1 (UCP1), proliferator-activated receptor gamma coactivator 1-alpha (PGC-1 $\alpha$ ), and AMP-activated protein kinase (AMPK) activity in brown preadipocyte treated with DE (50 and $100 \mu \mathrm{g} / \mathrm{mL}$ ) or vehicle (control) for 24 hours. $\beta$-Actin serves as a loading control. (C) Number of mitochondria were visualized by staining with MitoTracker Red 580 (red fluorescence) followed by treating with and without DE $(100 \mu \mathrm{g} / \mathrm{mL}, 24$ hours) and tumor necrosis factor- $\alpha$ (TNF- $\alpha ; 100 \mathrm{ng} / \mathrm{mL}, 1$ hour) in brown preadipocyte. Bars, $20 \mu \mathrm{m}$. (D) The effect of DE on intracellular reactive oxygen species (ROS). Synchronized brown preadipocytes were pretreated with DE (50 and $100 \mu \mathrm{g} / \mathrm{mL}, 24$ hours). CM- $\mathrm{H}_{2} \mathrm{DCF}-\mathrm{DA}$ emission was recorded using a fluorescence cytometer. The experiments were performed in triplicate and results are expressed as the mean \pm standard error of mean. p-AMPK, phosphorylated AMPK. ${ }^{\mathrm{a}} P<0.05$ and ${ }^{\mathrm{b}} P<0.01$ statistically significant differences between vehicle and DE groups.

\section{Danshen extracts protect against HFD-induced obesity and fatty liver}

After confirming that DE enhances mitochondrial function by induction of genes associated with key thermogenesis related gene, we next studied the effect of DE on weight gain in HF-induced mice. For this study, $\mathrm{LDLR}^{-/}$mice were provided with either a HFD ( $60 \%$ of calories from fat, HF) or a HF diet supplemented with DE (50 mg/kg of DE in HF diet, HF+DE) for 17 weeks (Fig. $3 \mathrm{~A})$. The mice fed the HF diet steadily gained weight up to 11 weeks compared to the normal group, but the DE supplemented group showed a significant decrease in weight gain from 9 weeks (HF vs. $\mathrm{HF}+\mathrm{DE}: 9$ weeks, $\Delta 11.72 \pm 0.61 \mathrm{~g}$ vs. $\Delta 7.825 \pm 0.883 \mathrm{~g}$;
11 weeks, $15.32 \pm 0.65$ g vs. $9.92 \pm 2.57 \mathrm{~g} ; 17$ weeks, $13.05 \pm 0.48$ g vs. $9.7 \pm 1.41 \mathrm{~g})$. Therefore, DE increases the thermogenesis by upregulating UCP1 expression in BAT, which is thought to have the effect of weight loss in HFD-induced mice.

To determine the effect of DE on blood glucose, a diabetic animal model was made using the STZ and an OGTT was performed (Fig. 3B). After oral administration of $2 \mathrm{mg} / \mathrm{kg}$ glucose, it was confirmed that the blood glucose significantly decreased in the DE-treated group from 30 minutes. Therefore, it was confirmed that DE was effective in lowering blood glucose level.

At 17 weeks of age, each experimental group was sacrificed and the liver histology was observed through H\&E staining 
0

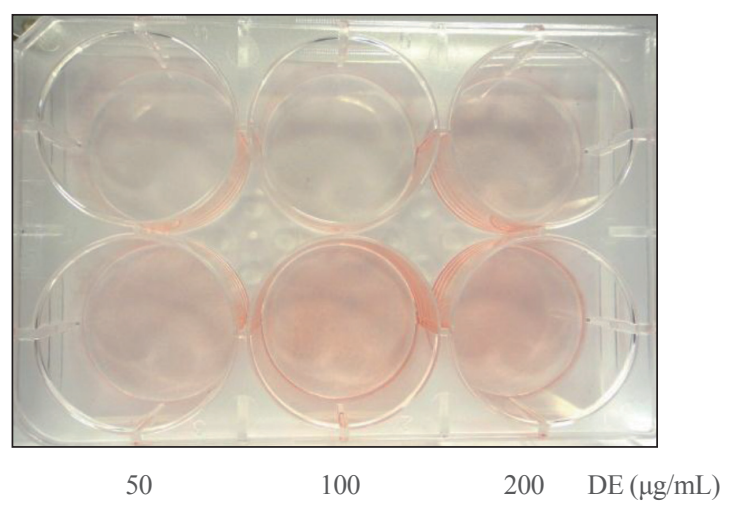

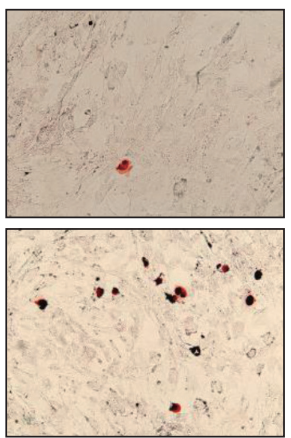

50
10

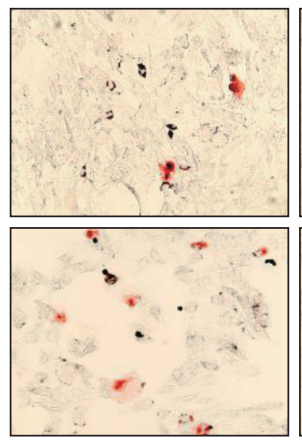

100
25

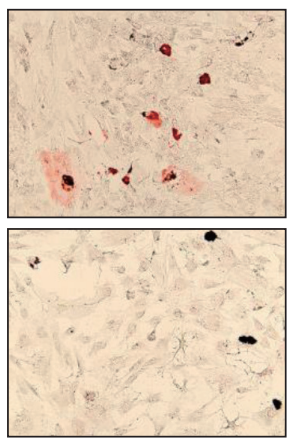

$200 \quad \mathrm{DE}(\mu \mathrm{g} / \mathrm{mL})$

$\begin{array}{llll}0 & 50 & 100 & \mathrm{DE}(\mu \mathrm{g} / \mathrm{mL})\end{array}$
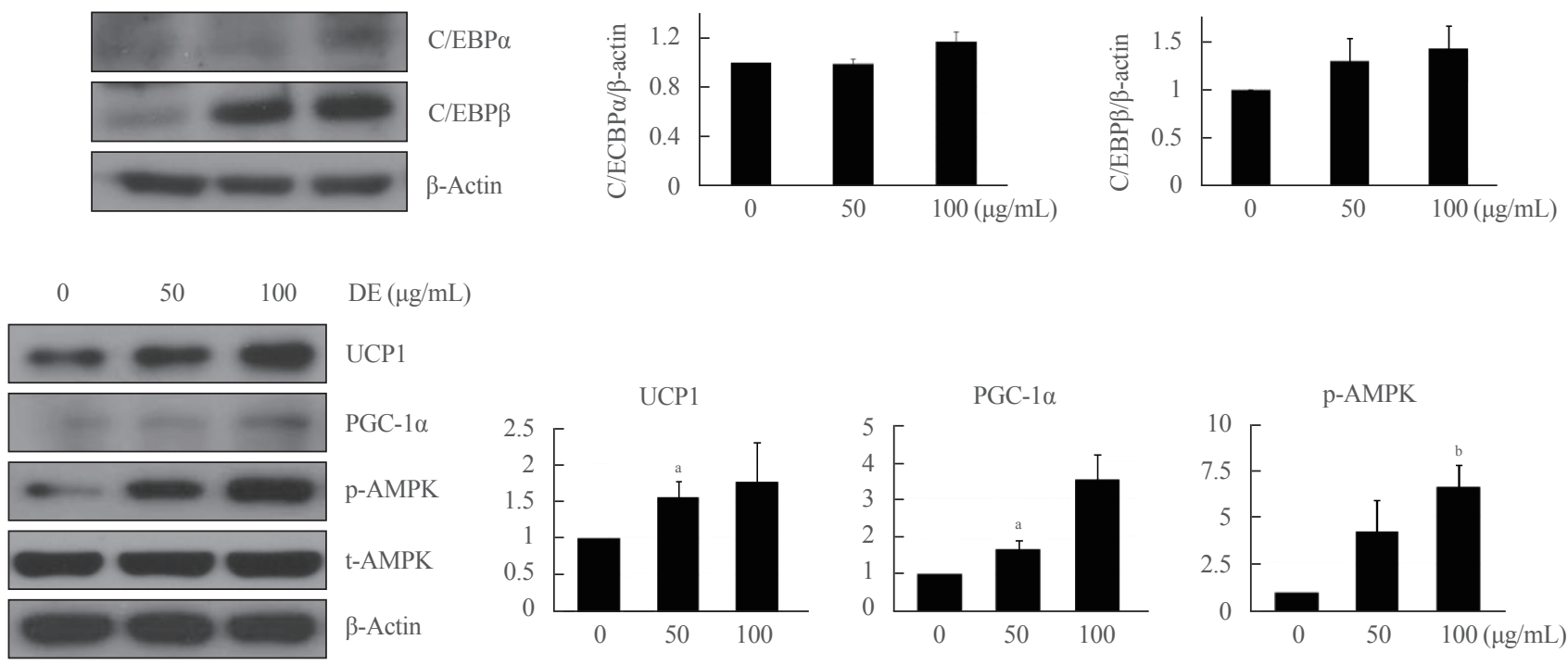

C

Fig. 2. Danshen extracts (DEs) promotes the brown adipocyte differentiation and mitochondrial functions in brown adipocytes. (A) After induction of adipogenesis for 10 days in brown preadipocytes treated with DE (10, 25, 50, 100, and $200 \mu \mathrm{g} / \mathrm{mL})$ or vehicle (control) for 7 days, Oil Red O staining was performed. Images were obtained with optical microscope at $\times 200$ magnification. (B) Western blot analysis of CCAAT/enhancer binding protein $(\mathrm{C} / \mathrm{EBP}) \alpha$ and $\mathrm{C} / \mathrm{EBP} \beta$ after induction of adipogenesis of brown preadipocytes for 10 days and treatment with DE (50 and $100 \mu \mathrm{g} / \mathrm{mL}$ ) or vehicle (control) for 24 hours. (C) Western blotting analysis of uncoupling protein 1 (UCP1), proliferatoractivated receptor gamma coactivator 1-alpha (PGC-1 $\alpha$ ), and AMP-activated protein kinase (AMPK) activity after induction of adipogenesis of brown preadipocytes for 10 days and treatment with DE (50 and $100 \mu \mathrm{g} / \mathrm{mL}$ ) or vehicle (control) for 24 hours. $\beta$-Actin serves as a loading control. Total-AMPK (t-AMPK) acts as a loading control for phosphorylated AMPK (p-AMPK). The experiments were performed in triplicate and results are expressed as the mean \pm standard error of mean. ${ }^{a} P<0.05$ and ${ }^{\mathrm{b}} P<0.005$ statistically significant differences between vehicle and DE (50 and $100 \mu \mathrm{g} / \mathrm{mL})$ groups.

(Fig. 3C). While the number of lipid-containing vesicles was significantly increased in the liver of the HF experimental group, it was not observed in the liver tissues of the DE supplemented group. As with the histological results, PPAR $\gamma$ and AMPK activity in liver tissue were observed using Western blot analysis, and as a result, there was a significant decrease in the group supplemented with DE. Therefore, DE increases heat production from brown fat tissue, thereby reducing weight and preventing fatty liver caused by a HFD.

\section{Influence of Danshen extracts on BAT and WAT metabolism}

To investigate the role of DE in BAT, we observed the morphology of brown fat tissue and key genes involved in BAT metabolism in experimental mice at 17 weeks. Multilocular brown adipocytes were observed in BAT from DE-treated mice, whereas 

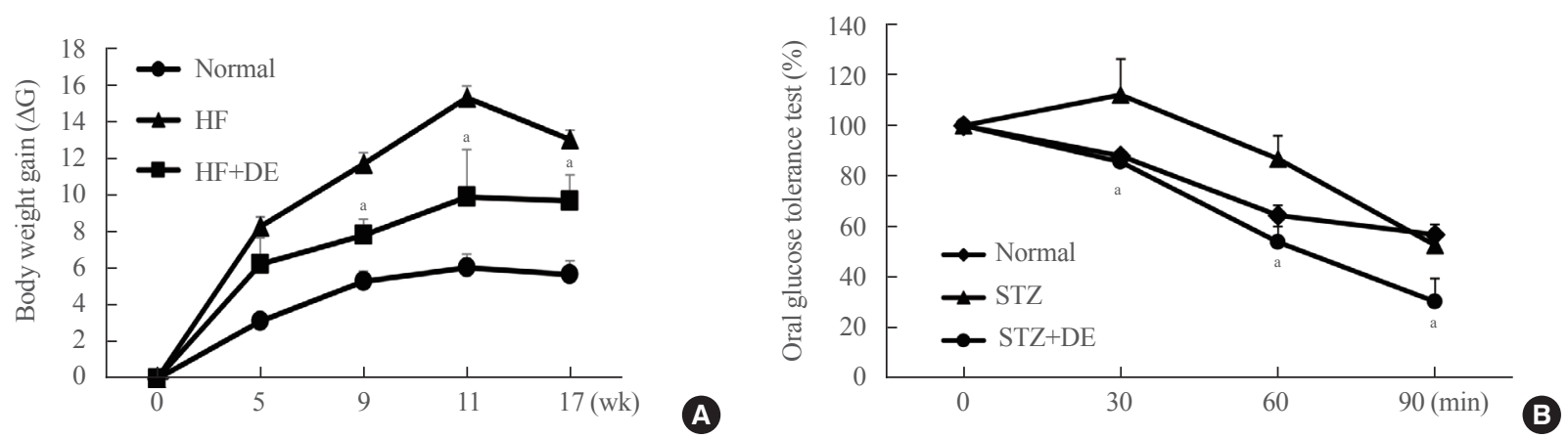

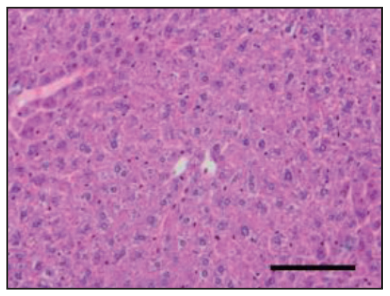

Normal

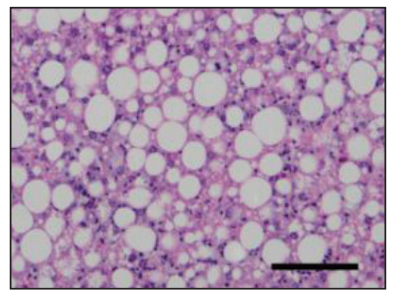

$\mathrm{HF}$

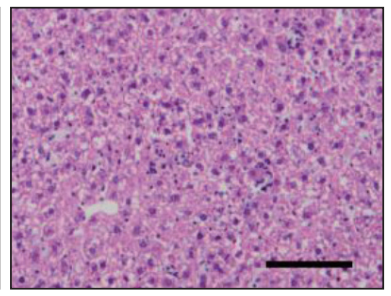

$\mathrm{HF}+\mathrm{DE}$
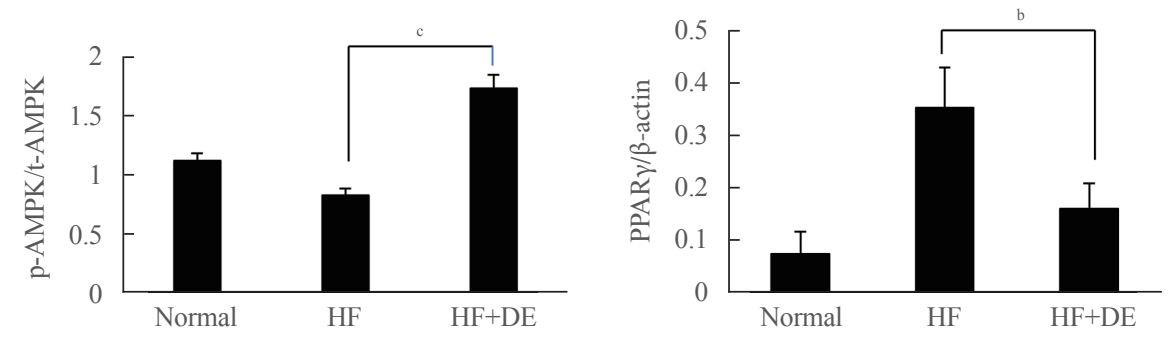

Fig. 3. Danshen extracts (DEs) reduces weight gain, blood glucose and fatty liver. (A) DE was administrated orally (50 mg/kg on daily) during 17 weeks; Calorie composition of dietary fat (w/wo $0.2 \%$ of DE) was described in Methods (high fat [HF]-diet; $60 \%$ of calories from fat). Number of animals in each experimental group of animals was five and starting weight was average of $25 \mathrm{~g}$. Weight measurements were performed once a week and indicated the average of weight gain $(\Delta \mathrm{G}) \pm$ standard deviation. (B) Streptozotocin (STZ)-induced diabetic mice, DE supplementation significantly decreased blood glucose compared to the STZ+sodium citrate (pH 4.5). (C) DE ameliorate non-alcoholic fatty liver disease through upregulating AMP-activated protein kinase (AMPK) activation in HF fed mice. H\&E staining and Western blotting analysis of peroxisome proliferator-activated receptor $\gamma(\mathrm{PPAR} \gamma)$ and AMPK activity in the liver tissue of experimental animals. Images were obtained with optical microscope at $\times 200$ magnification. p-AMPK, phosphorylated AMPK; t-AMPK, total AMPK; HF+DE, high fat diet supplemented with $0.2 \%$ DE. ${ }^{\text {a }} P<0.05$ statistically significant differences between $\mathrm{HF}$ group vs. $\mathrm{HF}+\mathrm{DE}$ groups; ${ }^{\mathrm{b}} P<0.01 ;{ }^{\mathrm{c}} P<0.005$.

lipid vesicle formation was observed in mice fed a HFD (Fig. 4A). Notably, mice fed a HFD displayed large unilocular fat droplets and outstanding decreases of more than $50 \%$ in interscapular brown adipocyte number compared with normal mice. Because BAT is known to play an important role in controlling the storage of total body fat and its mitochondria has a central role in energy consumption, we examined the key proteins involved in BAT metabolism in interscapular BAT (Fig. 4B). Expression of UCP1 protein was significantly increased more than two times in the BAT of DE-treated mice, but no difference in UCP1 expression was observed between the normal and HF diet groups. These data indicate that DE enhanced the functions of thermogenesis by upregulating UCP1.

The expression of PPAR $\gamma$ was significantly reduced in the BAT of HF diet groups $(P<0.01$ vs. normal ctrl), although its protein expression was increased by treatment with DE. Interestingly, DE also caused a significant increase in the expression of mitochondrial biogenesis-related markers, such as PGC-1 $\alpha$ and p-AMPK (increasing them more than 1.5 and 2 times, respectively, compared to mice fed an HF diet) in BAT. These data indicate that DE increases the induction of key molecules to determine brown fat fate and improves the mitochondrial function.

In addition, the effect of $\mathrm{DE}$ on the metabolism of white fat 


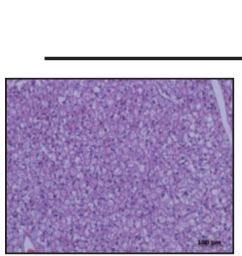

Normal
Brown fat tissue

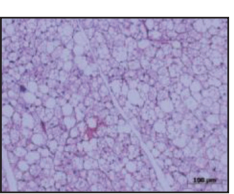

$\mathrm{HF}$

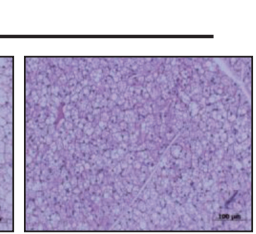

$\mathrm{HF}+\mathrm{DE}$

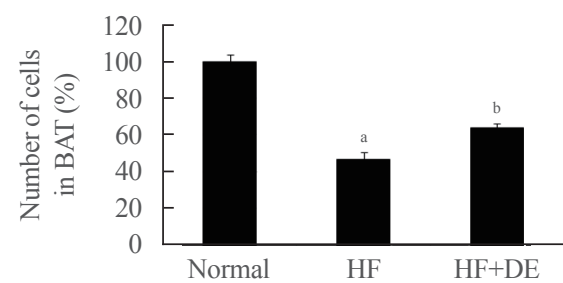

A
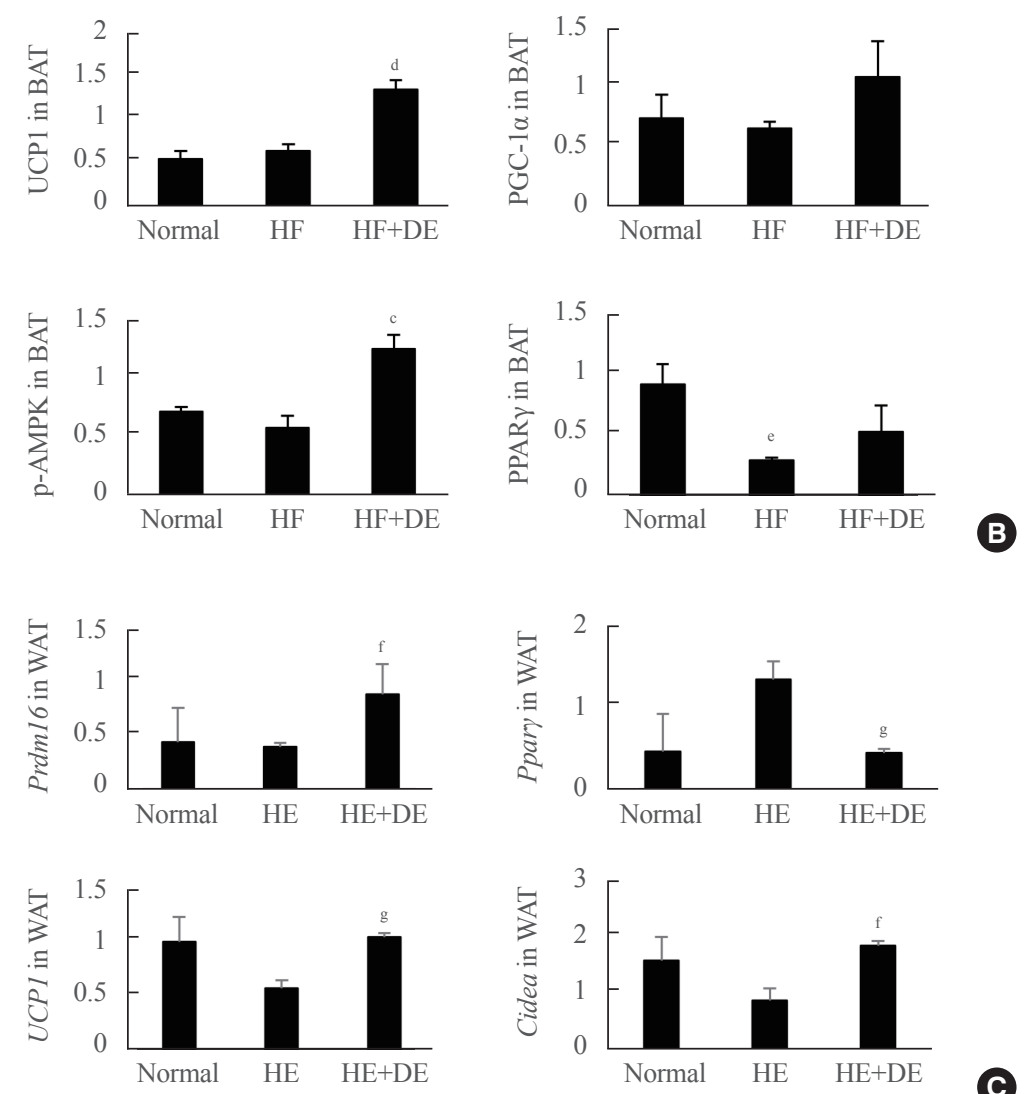

C

Fig. 4. Danshen extracts (DEs) activate brown adipose tissue (BAT) metabolism and browning of white adipose tissue (WAT) in obese mice. (A) H\&E staining of BAT from each experiment groups. Scale bars, $100 \mu \mathrm{m}$. The changes in number of cells of BAT was also measured. (B) Protein levels in interscapular BAT of key genes involved in BAT metabolism were analysis by using the Western blotting. $\beta$-Actin serves as a loading control. All animal experiments were measured at 17 weeks in obese mice with or without $0.2 \%$ of DE supplement. (C) Analysis of mRNA expression of key genes involved in browning of WAT. UCP1, uncoupling protein 1; PGC-1 $\alpha$, proliferator-activated receptor gamma coactivator 1-alpha; p-AMPK, phosphorylated AMP-activated protein kinase; PPAR $\gamma$, peroxisome proliferator-activated receptor $\gamma$; HF, high fat; Prdm16, PR domain containing 16. ${ }^{\mathrm{a}} P<0.005$ vs. normal; ${ }^{\mathrm{b}} P<0.01 \mathrm{vs}$. HF; ${ }^{\mathrm{c}} P<0.01,{ }^{\mathrm{d}} P<0.001$ statistically significant differences between $\mathrm{HF}$ group vs. HF+DE groups; ${ }^{\mathrm{e}} P<0.05$ vs. normal; ${ }^{\mathrm{f}} P<0.05$ and ${ }^{\mathrm{g}} P<0.01$ vs. HF.

was observed (Fig. 4C). First, it was confirmed that the expression of PPAR $\gamma$ was significantly reduced in the WAT of the experimental animals treated with DE. The browning from white fat to brown or beige fat has an effective effect on body fat burning potential for obesity treatment, so we observed whether DE is effective for the process of 'browning' WAT. The mRNA expression of gene related the browning, such as Prdm16, Ucp1, and Cidea was significantly upregulated by DE supplementa- tion in HFD-fed obese mice. In particular, Cidea is known to transcriptionally regulate UCP1 in fat cells for thermogenesis, which increased more than twice in the group treated with DE.

\section{DISCUSSION}

Danshen, the dried root of $S$. miltiorrhiza, is a commonly used traditional Chinese medicine for improving body function that 
is relevant in cardiovascular diseases, coronary heart disease, hyperlipidemia, and cerebrovascular disease [23]. Many studies have reported on the efficacy of Danshen in several metabolic disorders, and although Danshen has no major side effects, it may interact with other drugs and cause serious complications [24]. Although many studies have been reported on the lipidsoluble constituents of DEs such as tansinone IIA, the effect of water-soluble DE on obesity caused by BAT dysfunction has not yet been evaluated.

Our data demonstrated that water-soluble DE prevents HF-induced obesity and increases thermogenic activity by inducing UCP1 in BAT and WAT. As shown Fig. 4A, HFD-fed mice were displayed large unilocular fat droplets and outstanding decreases of more than $50 \%$ in interscapular brown adipocyte number compared with normal mice and DE enhanced brown adipocyte number. This data indicates that DE activated BAT function, enhancing cell proliferation, differentiation, and expression of the brown fat-specific UCP1. BAT contains a large amount of mitochondria with tissue-specific expression of the UCP1 protein [25], which oxidizes fat to generate heat and in this process the expression of UCP1 increases. Depletion of UCP1 leads to obesity, so UCP1 plays an important role in energy expenditure [26-28].

Upregulation of UCP1, which is closely associated with increased adaptive thermogenic activity and energy expenditure, is widely used as a marker not only of BAT activity, but also of a brown-like phenotype, such as that of beige adipocytes [11]. PGC-1 $\alpha$ plays a central role in the regulation of cellular energy metabolism. PGC-1 $\alpha$ is intimately involved in disorders such as obesity and diabetes and stimulates mitochondrial biogenesis to adaptive thermogenesis [10]. In brown preadipocytes, DE enhanced the PGC-1 $\alpha$, but not UCP1 expression, indicating that $\mathrm{DE}$ stimulates mitochondrial biogenesis by upregulating PGC$1 \alpha$ in preadipocytes and increases UCP1 expression during differentiation into mature brown adipocytes. The number of live mitochondria was also increased, consistent with the results of the treatment of DE increasing mitochondrial biogenesis in brown preadipocytes. TNF- $\alpha$ exposure causes decreased mitochondrial respiration, resulting in mitochondrial dysfunction. The number of mitochondria decreased due to TNF- $\alpha$ exposure to brown preadipocytes was recovered by treatment with DE. Therefore, DE is thought to improve mitochondrial dysfunction. ROS-like stress in adipocytes induces heat generation through UCP1 and mitochondrial biogenesis [29]. In brown preadipocytes, ROS production increases with the concentration of DE, so DE plays a key role in thermogenesis through UCP1 and mi- tochondrial production. As the concentration of DE increased, differentiation of brown preadipocyte into mature brown adipocytes increased, and expression of genes related to mitochondrial activity, such as UCP1, PGC1- $\alpha$, and AMPK was also increased.

To study the role of water-soluble DE on BAT mitochondrial function in obese animal models, $\mathrm{LDLR}^{-/}$mice were treated with a HFD. The purpose of this study was to observe changes in BAT and WAT when DE was administered using $\mathrm{LDLR}^{-/}$ mice, which can induce arteriosclerosis through a HFD. The body weight of the experimental animals that consumed the HFD steadily increased as expected, but did not increase from 17 weeks.

In this study, DE reduced the weight gain and fatty liver in HFD-fed obese mice and reduced blood glucose level in the STZ-induced diabetic model. In addition, it was confirmed that DE significantly reduced blood glucose level in the STZ-induced diabetes model through OGTT.

Dietary intake was not studied in this study, but it was confirmed that the expression of sirtuin-1 (Sirt1) and p-AMPK expression was increased in hypothalamus of experimental animals administered with DE. Key energy sensors, such as the AMPK or SIRT1, are essential in agouti-related peptide (AgRP) and proopiomelanocortin (POMC) cells to ensure proper energy balance. The result that the expression of AMPK or Sirt1 was increased in the hypothalamus of the DE-treated group is predicted to decrease dietary intake as the DE affects these neurons involved in food intake to some extent but there was no particular feature in feeding behavior.

Our data have shown that DE have thermogenic activity by inducing UCP1 in BAT and WAT in HFD-fed mice. Prdm16 is a transcriptional coregulatory responsible for browning induction and thermogenic maintenance of the beige adipocyte phenotype [30]. This study found that the presence of Prdm16 in subcutaneous WAT leads to a significant upregulation of brown fat selective genes UCP1, and Cidea. This upregulation lead to the development of a BAT-like phenotype within the WAT.

Upadhyay et al. [31] reported that BAT activation enhances oxygen consumption, resulting in increased energy expenditure per day [26,31]. It remains unclear whether DE treatment has a direct or indirect effect on oxygen consumption; however, one possibility is that DE increases UCP1 expression in both BAT and WAT, perhaps the increased UCP1 will enhance the thermogenesis and energy expenditure as reported in the previous study [32].

As shown in our data, many previous studies have reported that PGC- $1 \alpha$ interacts with a broad range of transcription factors 
involving in adaptive thermogenesis and mitochondrial biogenesis. PGC-1 $\alpha$ is expressed at high levels in tissues where mitochondria are abundant and oxidative metabolism is active, such as BAT [30,33].

The expression of PPAR $\gamma$ was significantly reduced in the BAT of HFD-fed groups, although its protein expression was increased by treatment with DE. These results are consistent with a previous report finding that PPAR $\gamma$ is required to maintain the thermogenic capacity of mature brown adipocytes, which can be induced by activating $\beta$-adrenergic signaling. They show that expression of the known PPAR $\gamma$ target Gyk correlates with human brown fat activity and identify Gyk as a partial mediator of PPAR $\gamma$ function. Taken together, DE is thought to affect $\beta$-adrenergic signaling-mediated induction of brown adipocytes by activating PPAR $\gamma$ in BAT.

Activation of AMPK by single ginseng increases the expression of mitochondrial biogenesis signals through PGC1 $\alpha$ expression. Our results indicate that DE-induced transcriptional activation of UCP1 can be attributed to enhanced phosphorylation of AMPK.

In summary, DE supplementation increased the expression of UCP1 protein in both WAT and BAT in HFD-fed obese mice, and this was accompanied by upregulation of mitochondrial thermogenic genes. As these results, DE relieved weight gain, fatty liver and blood sugar due to HFD. Therefore, Dietary DE may therefore be useful to protect against obesity and metabolic dysregulation.

\section{CONFLICTS OF INTEREST}

No potential conflict of interest relevant to this article was reported.

\section{ACKNOWLEDGMENTS}

This research was supported by a grant from the Korea Health Technology R\&D Project through the Korea Health Industry Development Institute (KHIDI), funded by the Ministry of Health \& Welfare, Republic of Korea (grant number: HR18C0012) and by Basic Science Research Program through the National Research Foundation of Korea (NRF) funded by the Ministry of Education (grant number: 2020R1I1A1A01074357).

\section{AUTHOR CONTRIBUTIONS}

Conception or design: Y.H.C., E.J.L. Acquisition, analysis, or interpretation of data: Y.H.C., C.R.K., H.J.L., Y.S.C., E.J.L. Drafting the work or revising: Y.H.C. Final approval of the manuscript: Y.H.C., E.J.L.

\section{ORCID}

Yoon Hee Cho https://orcid.org/0000-0002-0731-8439

Eun Jig Lee https://orcid.org/0000-0003-3231-9887

\section{REFERENCES}

1. Hossain P, Kawar B, El Nahas M. Obesity and diabetes in the developing world: a growing challenge. N Engl J Med 2007; 356:213-5.

2. Lazar MA. How obesity causes diabetes: not a tall tale. Science 2005;307:373-5.

3. Cannon B, Nedergaard J. Brown adipose tissue: function and physiological significance. Physiol Rev 2004;84:277359.

4. Himms-Hagen J. Brown adipose tissue thermogenesis: interdisciplinary studies. FASEB J 1990;4:2890-8.

5. Dulloo AG, Miller DS. Energy balance following sympathetic denervation of brown adipose tissue. Can J Physiol Pharmacol 1984;62:235-40.

6. Petersen KF, Dufour S, Befroy D, Garcia R, Shulman GI. Impaired mitochondrial activity in the insulin-resistant offspring of patients with type 2 diabetes. N Engl J Med 2004; 350:664-71.

7. Puigserver $\mathrm{P}, \mathrm{Wu} Z$, Park CW, Graves R, Wright M, Spiegelman BM. A cold-inducible coactivator of nuclear receptors linked to adaptive thermogenesis. Cell 1998;92:829-39.

8. Uldry M, Yang W, St-Pierre J, Lin J, Seale P, Spiegelman BM. Complementary action of the PGC-1 coactivators in mitochondrial biogenesis and brown fat differentiation. Cell Metab 2006;3:333-41.

9. Bouillaud F, Ricquier D, Thibault J, Weissenbach J. Molecular approach to thermogenesis in brown adipose tissue: cDNA cloning of the mitochondrial uncoupling protein. Proc Natl Acad Sci U S A 1985;82:445-8.

10. Barbera MJ, Schluter A, Pedraza N, Iglesias R, Villarroya F, Giralt M. Peroxisome proliferator-activated receptor alpha activates transcription of the brown fat uncoupling protein-1 gene: a link between regulation of the thermogenic and lipid oxidation pathways in the brown fat cell. J Biol Chem 2001; 276:1486-93.

11. Lin J, Wu PH, Tarr PT, Lindenberg KS, St-Pierre J, Zhang 
CY, et al. Defects in adaptive energy metabolism with CNSlinked hyperactivity in PGC-1alpha null mice. Cell 2004; 119:121-35.

12. Canto C, Auwerx J. PGC-1alpha, SIRT1 and AMPK, an energy sensing network that controls energy expenditure. Curr Opin Lipidol 2009;20:98-105.

13. Zong H, Ren JM, Young LH, Pypaert M, Mu J, Birnbaum MJ, et al. AMP kinase is required for mitochondrial biogenesis in skeletal muscle in response to chronic energy deprivation. Proc Natl Acad Sci U S A 2002;99:15983-7.

14. Huypens P, Quartier E, Pipeleers D, Van de Casteele M. Metformin reduces adiponectin protein expression and release in 3T3-L1 adipocytes involving activation of AMP activated protein kinase. Eur J Pharmacol 2005;518:90-5.

15. Farmer SR. Obesity: be cool, lose weight. Nature 2009;458: $839-40$.

16. Cho YH, Ku CR, Hong ZY, Heo JH, Kim EH, Choi DH, et al. Therapeutic effects of water soluble danshen extracts on atherosclerosis. Evid Based Complement Alternat Med 2013; 2013:623639.

17. Han JY, Fan JY, Horie Y, Miura S, Cui DH, Ishii H, et al. Ameliorating effects of compounds derived from Salvia miltiorrhiza root extract on microcirculatory disturbance and target organ injury by ischemia and reperfusion. Pharmacol Ther 2008;117:280-95.

18. Shiao MS, Chiu JJ, Chang BW, Wang J, Jen WP, Wu YJ, et al. In search of antioxidants and anti-atherosclerotic agents from herbal medicines. Biofactors 2008;34:147-57.

19. Klein J, Fasshauer M, Ito M, Lowell BB, Benito M, Kahn CR. Beta(3)-adrenergic stimulation differentially inhibits insulin signaling and decreases insulin-induced glucose uptake in brown adipocytes. J Biol Chem 1999;274:34795-802.

20. Miranda S, Gonzalez-Rodriguez A, Revuelta-Cervantes J, Rondinone CM, Valverde AM. Beneficial effects of PTP1B deficiency on brown adipocyte differentiation and protection against apoptosis induced by pro- and anti-inflammatory stimuli. Cell Signal 2010;22:645-59.

21. Hong JM, Park CS, Nam-Goong IS, Kim YS, Lee JC, Han MW, et al. Curcumin enhances docetaxel-induced apoptosis of 8505C anaplastic thyroid carcinoma cells. Endocrinol Metab (Seoul) 2014;29:54-61.

22. Tedesco L, Valerio A, Cervino C, Cardile A, Pagano C, Vet- tor R, et al. Cannabinoid type 1 receptor blockade promotes mitochondrial biogenesis through endothelial nitric oxide synthase expression in white adipocytes. Diabetes 2008; 57:2028-36.

23. Zhou L, Zuo Z, Chow MS. Danshen: an overview of its chemistry, pharmacology, pharmacokinetics, and clinical use. J Clin Pharmacol 2005;45:1345-59.

24. Cheng TO. Cardiovascular effects of Danshen. Int J Cardiol 2007;121:9-22.

25. Kelly LJ, Vicario PP, Thompson GM, Candelore MR, Doebber TW, Ventre J, et al. Peroxisome proliferator-activated receptors gamma and alpha mediate in vivo regulation of uncoupling protein (UCP1, UCP-2, UCP-3) gene expression. Endocrinology 1998;139:4920-7.

26. Palou A, Pico C, Bonet ML, Oliver P. The uncoupling protein, thermogenin. Int J Biochem Cell Biol 1998;30:7-11.

27. Wolf G. Brown adipose tissue: the molecular mechanism of its formation. Nutr Rev 2009;67:167-71.

28. Feldmann HM, Golozoubova V, Cannon B, Nedergaard J. UCP1 ablation induces obesity and abolishes diet-induced thermogenesis in mice exempt from thermal stress by living at thermoneutrality. Cell Metab 2009;9:203-9.

29. Ortega SP, Chouchani ET, Boudina S. Stress turns on the heat: regulation of mitochondrial biogenesis and UCP1 by ROS in adipocytes. Adipocyte 2017;6:56-61.

30. Evans D, Minouchehr S, Hagemann G, Mann WA, Wendt D, Wolf A, et al. Frequency of and interaction between polymorphisms in the beta3-adrenergic receptor and in uncoupling proteins 1 and 2 and obesity in Germans. Int J Obes Relat Metab Disord 2000;24:1239-45.

31. Upadhyay SK, Eckel-Mahan KL, Mirbolooki MR, Tjong I, Griffey SM, Schmunk G, et al. Selective Kv1.3 channel blocker as therapeutic for obesity and insulin resistance. Proc Natl Acad Sci U S A 2013;110:E2239-48.

32. Matthias A, Ohlson KB, Fredriksson JM, Jacobsson A, Nedergaard J, Cannon B. Thermogenic responses in brown fat cells are fully UCP1-dependent. UCP2 or UCP3 do not substitute for UCP1 in adrenergically or fatty scid-induced thermogenesis. J Biol Chem 2000;275:25073-81.

33. Liang H, Ward WF. PGC-1alpha: a key regulator of energy metabolism. Adv Physiol Educ 2006;30:145-51. 